\title{
Colloid particle transport in a microcapillary: NMR study of particle and suspending fluid dynamics
}

\section{Authors: Einar O. Fridjonsson \& Joseph D. Seymour.}

NOTICE: this is the author's version of a work that was accepted for publication in Chemical Engineering Science. Changes resulting from the publishing process, such as peer review, editing, corrections, structural formatting, and other quality control mechanisms may not be reflected in this document. Changes may have been made to this work since it was submitted for publication. A definitive version was subsequently published in Chemical Engineering Science, 153, October 2016, DOI\#10.1016/j.ces.2016.07.005.

Fridjonsson EO, Seymour JD "Colloid particle transport in a microcapillary: NMR study of particle and suspending fluid dynamics," Chemical Engineering Science, 2016 October 22; 153: 165-173. 


\title{
Colloid particle transport in a microcapillary: NMR study of particle and suspending fluid dynamics
}

\author{
Einar O. Fridjonsson ${ }^{a, b, n}$, Joseph D. Seymour ${ }^{a}$ \\ a Department of Chemical and Biological Engineering, Montana State University, Bozeman, MT, USA \\ b School of Mechanical and Chemical Engineering, The University of Western Australia, Crawley, WA 6009, Australia
}

$\begin{array}{llllllllll} & \text { I } & \text { G } & \text { H } & \text { L } & \text { I } & \text { G } & \text { H } & \text { T } & \text { S }\end{array}$

- NMR 'active' colloidal particles at 22 vol\% flowed through a $\mu$-capillary

- Simultaneous measurement of particle and suspending fluid phase dynamics

- Dynamics of suspending fluid contains particle structure information

- Shear induced migration within $\mu$-capillaries

- Results provide experimental data for further investigation and model verification

K E Y W O R D S

A B S T R A C T

Microcapillary

Colloids

NMR

Hydrodynamic

dispersion Particle

dynamics

\begin{abstract}
Precise manipulation of the hydrodynamic interaction between particles is particularly important for operation of microfluidic devices. Shear-induced migration gives rise to dynamical patterns within the flow that have been observed in a range of systems. In this work NMR 'active' colloidal particles (a $\left.1 \frac{1}{4} 1.25 \mathrm{~mm}\right)$ at volume fraction of $22 \%$ in an aqueous phase are flowed through a m-capillary $\left(\mathrm{R}^{1 / 4} 126 \mathrm{~mm}\right)$ and the transport dynamics of the particle and suspending fluid phases are studied using dynamic NMR techniques. Simultaneous interrogation of shear rheology of the suspending fluid and particle phases of colloidal suspensions is presented. The dynamic behavior of the suspending fluid is shown to carry within it information about the structure of the colloidal particle ensembles on the time scales in-vestigated $(\Delta 1 / 425$ $\mathrm{ms}-250 \mathrm{~ms}$ ) providing rich experimental data for further investigation and model verification. The importance of determining the particle concentration profile within $\mu$-capillaries is explicitly demonstrated as shear induced migration causes significant concentration gradients to occur at
\end{abstract}

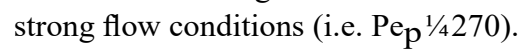

I N T R O D U C T I O N

The transport dynamics of colloid particles (diameter (2a):

$1 \mathrm{~nm}$ to $10 \mu \mathrm{m}$ (Russel, 1981)) suspended in a liquid, is important in many industrial and environmental processes and has therefore received significant attention in the scientific literature (Brady and Vicic, 1995, Vermant and Solomon, 2005). Precise manipulation of the hydrodynamic interaction between particles is important for operation of microfluidic devices (Stone et al., 2004); shear in-duced migration of the suspended particles being particularly important (Reddig and Stark, 2013). Microfluidic applications of shear induced migration include generation and manipulation of emulsion droplets (Christopher and Anna, 2007), polystyrene microspheres (Sochol et al., 2011) and biological cells (Yun et al., 2013). Flow induced migration gives rise to dynamical patterns which have been observed in a range of systems. These include coiling of semi-flexible polymers (Chelakkot et al., 2012, Harasim et al., 2013), anomalous crystal phonon modes (Beatus et al., 2007), collective dynamics (Janssen et al., 2012), inertial migration (Di Carlo et al., 2009) and shear induced migration (Semwogerere et al., 2007, Snijkers et al., 2013).

An important example is the Segre-Silberberg effect caused by inertial forces acting on the particles in non-zero Reynolds number (Re) flow (Leal, 1980) where rigid spheres assemble on an annulus at approximately 0.6 of the internal radii of a circular tube during laminar flow (Segre and Silberberg, 1962). When non-zero Re bounded shear flow is introduced to sphere trajectories a rich class of solutions is observed (Subramanian and Koch, 2006), while at non-inertial conditions a threshold for irreversibility and chaos is 
observed in many body particle suspensions where time reversible equations of motion should govern (Pine et al., 2005).

Forces which determine the particle dynamics are hydrodynamic, Brownian and interparticle (Brady, 1993). The hydrodynamic forces are due to the presence of fluid between particles which allows the propagation of particle interactions including those due to the application of a linear external shear field. Brownian forces are due to the stochastic motion of the suspending fluid molecules impacting the suspended particle. In colloidal suspensions, the particles in a suspending fluid are small enough that Brownian forces affect their dynamics (Brown 1828; Einstein 1906; Smoluchowski, 1906). Interparticle forces can be divided into those due to the excluded volume repulsion, electrostatic interaction, van der Waals forces (short-range and attractive), entropic forces and steric forces (either steric repulsion or attractive depletion) (Russel et al., 1989). If a colloidal suspension is unstable it can flocculate, i.e. form aggregates of colloidal particles due to the removal of the electrostatic barrier separating particles, due to addition of flocculants (charged polymer), depletants (non-adsorbed polymers) or colloid deformation (Russel et al., 1989). Mono-disperse hard-sphere colloidal suspensions are the simplest type of colloidal suspension, where the particles interact through hydrodynamic and Brownian forces, and can be fully characterized by the volume fraction $(\phi)$ and the particle Péclet number $\left(P e_{\mathrm{p}}\right)$ (Brady and Vicic, 1995). They can also be used to form the building blocks for understanding more complex colloid suspensions (Brady, 1996, Yurkovetsky and Morris, 2008).

Detailed experimental, analytical and simulation studies have been conducted to investigate the behavior of hard sphere colloidal suspensions over the full range of volume fraction $(\phi)$ from dilute to close packed and for $P e_{p}$ numbers encompassing the rheological behavior from weak flow conditions $\left(P e_{p} \sim 1\right)$ to strong flow conditions $\left(P e_{p}>>1\right)$ (Bergenholtz et al., 2002, Foss and Brady, 2000). In this paper, we focus on the dynamics in the shear flow dominated regime $\left(P e_{p}>>1\right)$ for a $\phi=0.22$ hard sphere suspension (Wassenius et al., 2003), where $P e_{p}$ and $\phi$ are defined as:

$P e_{p}=\frac{6 \pi \eta_{0}\langle\dot{\gamma}\rangle a^{3}}{k_{B} T}$

$\phi=\frac{4 \pi a^{3} n}{3}$

Here $\eta_{0}$ is the viscosity of the suspending fluid, $\langle\dot{\gamma}\rangle$ is the averaged shear rate, $a$ is the mean radius of the colloidal spheres, $k_{B}$ is the Boltzmann constant, $T$ is the absolute temperature and $n$ is the number density. The averaged shear rate is defined as $\langle\dot{\gamma}\rangle=\frac{4}{3} \frac{v_{m}}{R}$, where $v_{m}$ is the maximum velocity in the $\mu$-capillary and $R$ is the radius of the $\mu$-capillary. Additional dimensionless numbers used to characterise the flow are the tube Péclet number $\left(P e_{t}\right)$, particle Reynolds number $\left(R e_{p}\right)$ and the tube Reynolds number $\left(R e_{t}\right)$ :

$P e_{t}=\frac{2 R\langle v\rangle}{D_{w}}$

$\operatorname{Re}_{p}=\frac{a^{2}\langle\dot{\gamma}\rangle \rho}{\eta_{0}}$

$R e_{t}=\frac{2 R\langle v\rangle \rho}{\eta_{0}}$

In which $\langle v\rangle$ is the average velocity in the capillary, $D_{w}$ is the diffusion coefficient of the suspending fluid and $\rho$ is the density of the suspending fluid in the capillary.
In this study pulsed gradient spin echo (PGSE) nuclear magnetic resonance (NMR) techniques are used to independently measure the transport properties of the suspending fluid phase and particle phase by using oil filled hard sphere core shell particles. These NMR "active" particles have previously been studied with NMR to measure the diffusion characteristics of the internal oil (Wassenius et al., 2003) and velocimetry (Wassenius and Callaghan, 2004, 2005). They have been used to demonstrate irreversibility of particle motion at dilute concentrations (Brown et al., 2007), shear induced migration in capillaries (Brown et al. 2009) and hydrodynamic dispersion in microcapillaries (Fridjonsson et al., 2014b). They have also been used to study deposition in porous media (Fridjonsson et al., 2014a), flow partitioning in bifurcations (Fridjonsson et al., 2011), effects of oscillatory flow (Evertz et al., 2012) and transport dynamics in open cell polymer foams (Brosten et al., 2010).

The stochastic motion of colloidal suspension particles can be characterized by the Stokes-Einstein-Sutherland diffusivity,

$D_{S E S}=\frac{k_{B} T}{6 \pi \eta_{0} a}$

Ideal hard-sphere colloids have short-range repulsive interparticle interactions and no long-range electrostatic interactions (Antl et al., 1986). To achieve hard-sphere colloidal interactions in experiments it is almost always required that the surface of the colloidal spheres be manipulated (Antl et al., 1986) and that some charge be present on the surface (Wassenius et al., 2003). For the colloidal particles used in this work the particles are charge stabilized by adding polyvinyl alcohol (PVA) to the suspending fluid which absorbs on the particle surface and generates short range repulsive forces. Further information on colloidal dynamics has been reviewed elsewhere (Russel et al., 1989, Stickel and Powell, 2005, Vermant and Solomon, 2005).

\section{Materials and methods}

NMR experiments were conducted using a Bruker $250 \mathrm{MHz}$ superconducting magnet, a Mirco5 probe base and magnetic field gradient coil with $\mathbf{g}_{\max }=1.7 \mathrm{~T} / \mathrm{m}$ in three orthogonal $(x, y, z)$ gradient directions. The flow setup is placed inside a $5 \mathrm{~mm}$ radiofrequency (rf) coil at a distance $>2000 R$ from the beginning of the microcapillary section of the flow setup so that all experiments are conducted on the steady state regime of colloidal migration due to shear induced migration (Semwogerere et al., 2007). To study the fluid dynamics of the colloidal suspension inside the capillary, spectrally resolved PGSE NMR techniques are used (Callaghan 1991, 2011; Price, 2009). These allow for the simultaneous independent investigation of the dynamics of both the colloid and suspending fluid phases of the suspensions (Brown et al., 2007). The dynamics are measured at $Q=0.25$ and $0.50 \mathrm{~mL} / \mathrm{h}$. In the current work a microcapillary with internal radius $(R)$ of $126 \mu \mathrm{m}$ is used. The flow conditions (see Table 1 ) are characterized as strong flow $(\mathrm{Pe}>>1$ ) with inertial forces small when compared with viscous forces $(\operatorname{Re}<1)$.

NMR can be used to measure the molecular motion of particular chemical species within a sample non-destructively by

Table

Dimensionless numbers $\left(P e_{p}, P e_{t}, R e_{p}, R e_{t}\right)$ characterizing the flow conditions studied in the $\mu$-capillary.

\begin{tabular}{lllll}
\hline $\boldsymbol{Q}(\mathbf{m L} / \mathbf{h})$ & $\boldsymbol{P e}$ & $P e_{\boldsymbol{t}}$ & $\boldsymbol{R e}_{\boldsymbol{p}}\left(\times \mathbf{1 0}^{\mathbf{5}}\right)$ & $\boldsymbol{R e}_{\boldsymbol{t}}$ \\
\hline 0.25 & 270 & 160 & 4.6 & 0.35 \\
0.50 & 540 & 321 & 9.3 & 0.70 \\
\hline
\end{tabular}


(a)

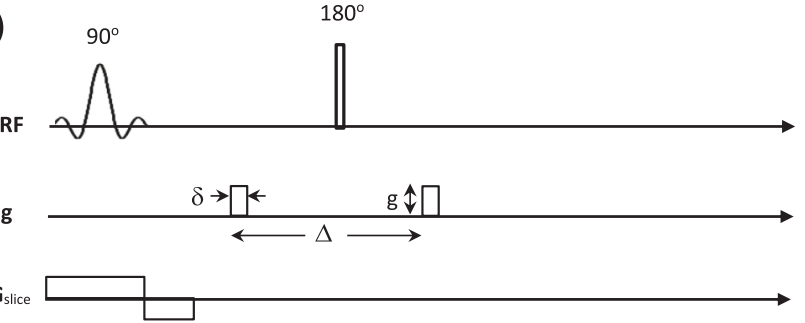

(b)

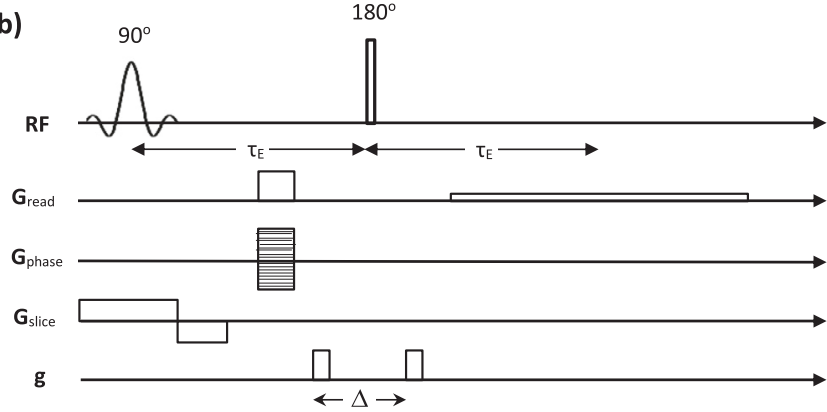

Fig. 1. Shows the NMR timing diagrams for (a) slice selective pulsed gradient spin echo (PGSE) pulse sequence, where $\Delta$ is the observation time, $\delta$ and $g$ are respectively the duration and magnitude of the pulsed gradients. NMR parameters used: Acquisition points (Acq_pts): 256, sweep width (SW): $5 \mathrm{kHz}$, gradient duration $(\delta)$ : $2 \mathrm{~ms}$, observation time $(\Delta): 25 \mathrm{~ms}$ to $500 \mathrm{~ms}$, number of gradient $(\mathrm{g})$ points: $128, \mathrm{~g}$ : $\left[-G_{\max }, 0,+G_{\max }\right] \mathrm{mT} / \mathrm{m}, G_{\max }$ : variable (dependent on $\Delta$ ), number of scans $\left(N_{s}\right)$ : 16 , repetition time $T_{R}: 2 \mathrm{~s}$, total experiment time $\left(T_{\text {exp }}\right): 1 \mathrm{~h} 8 \mathrm{~min}$. (b) Velocity map pulse sequence, showing a combined slice selective NMR imaging sequence with associated magnetic field gradients $\left(\mathbf{G}_{\text {read }}, \mathbf{G}_{\text {phase }}\right)$ and magnetic field gradients $(g)$ to measure displacement. NMR parameters used: Acquisition points (Acq_pts): 128 by 32, field of view (FOV): $1500 \mu \mathrm{m}$ by $375 \mu \mathrm{m}$, in-plane resolution: $11.7 \mu \mathrm{m}$ by $11.7 \mu \mathrm{m}$, slice thickness: $5 \mathrm{~mm}$, sweep width (SW): $100 \mathrm{kHz}$, gradient duration $(\delta)$ : $1 \mathrm{~ms}$, observation time $(\Delta): 20 \mathrm{~ms}$, number of gradient $(\mathrm{g})$ points: $2, \mathrm{~g}$ : $[50,125]$ $\mathrm{mT} / \mathrm{m}$, number of averages: 512 , repetition time $T_{R}: 1 \mathrm{~s}$, total experiment time $\left(T_{\exp }\right.$ ): 9 h 6 min.

applying magnetic field gradients across the sample. The most commonly used method is based on PGSE NMR techniques (Callaghan, 1991, 2011; Price, 2009). These techniques are based on the fact that there is a magnetic phase associated with the translational motion of a nucleus with a time dependent displacement

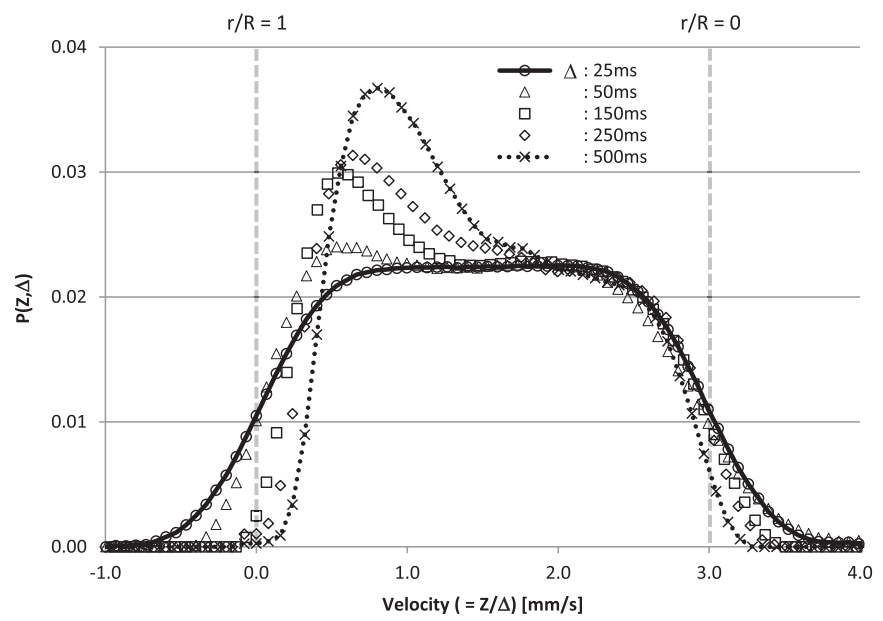

Fig. 3. The propagators of water in the axial-to-flow $(Z)$ direction at different experimental observation times $\Delta=25,50,150,250$ and $500 \mathrm{~ms}$ for $Q=0.25 \mathrm{~mL} / \mathrm{h}$. Observation time $(\Delta)$ evolution of the propagators is consistent with what has been previously observed in NMR experiments due to the combined effects of Taylor dispersion and capillary wall restriction (Codd et al. 1999). Dashed lines, for reference, indicate velocities consistent with the wall $(r / R=1)$ and center $(r / R=0)$ of the capillary. A hat-function shape is expected for Poiseuille flow at $\Delta=0$, the deviation at $\Delta=25 \mathrm{~ms}$ is due to molecular self-diffusion resulting in a shape which is the convolution of a hat-function due to Poiseuille flow and a Gaussian due to molecular diffusion. The higher probability of molecules at lower velocity as $\Delta$ increases is due to wall restriction, while the narrowing of the distributions is due to Taylor dispersion, the eventual shape will be a Gaussian as all molecules have sampled all streamlines $\left(\Delta>R^{2} / D_{0}\right)$.

$\mathbf{r}(t)$. Then the self-motion of a nuclear spin is described by the conditional probability $P\left(\mathbf{r} \mid \mathbf{r}^{\prime}, t\right)$, the probability that a nuclear spin originally at $\mathbf{r}$ will move to $\mathbf{r}^{\prime}$ over time $t$. In this work a PGSE pulse sequence (Fig. 1a - NMR parameters are shown in caption) is used to measure the displacement over varying times $\Delta$. In this pulse sequence a magnetic field gradient $\mathbf{g}$ of duration $\delta$, initially imparts a spatially dependent phase shift $\phi(\mathbf{r})$ on the spins,

$\phi(\mathbf{r})=\gamma \delta \mathbf{g} \cdot \mathbf{r}$

where $\gamma$ is the gyromagnetic ratio. After a time delay $\Delta$, the displacement observation time, the molecules will migrate to $\mathbf{r}^{\prime}$, and a second equal but oppositely-orientated gradient pulse is applied. (a)

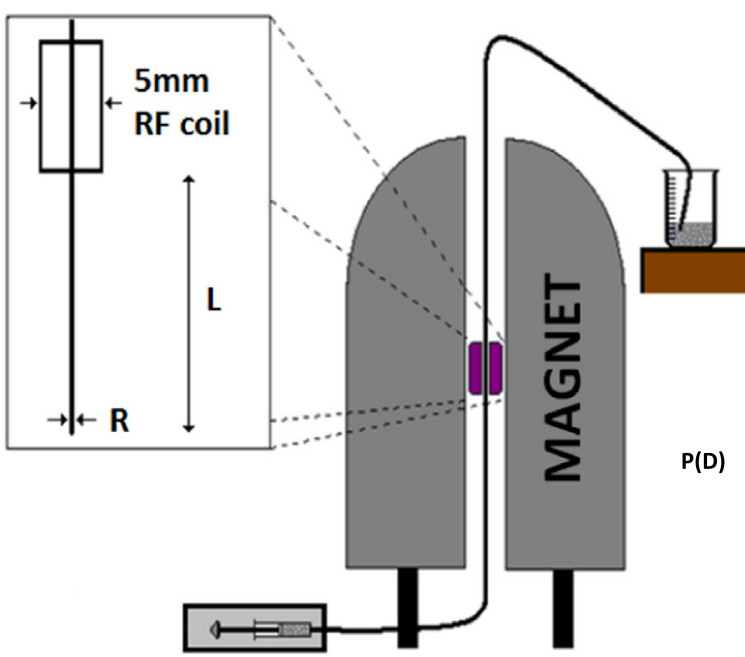

(b)
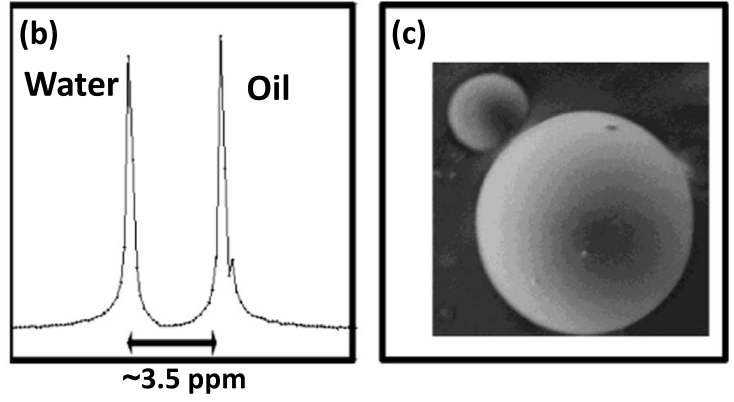

$0.2 \dashv$ (d)

0.1

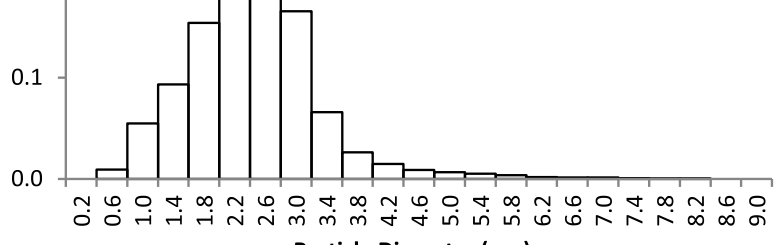

Particle Diameter $(\mu \mathrm{m})$

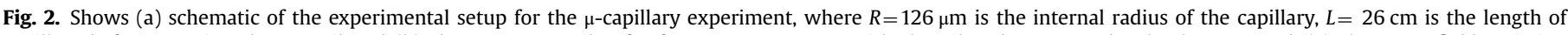

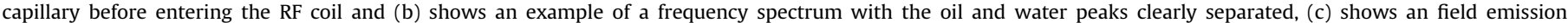
micrograph of two spherical particles, (d) shows the number based particle size distribution of the colloidal suspension used in this work. 

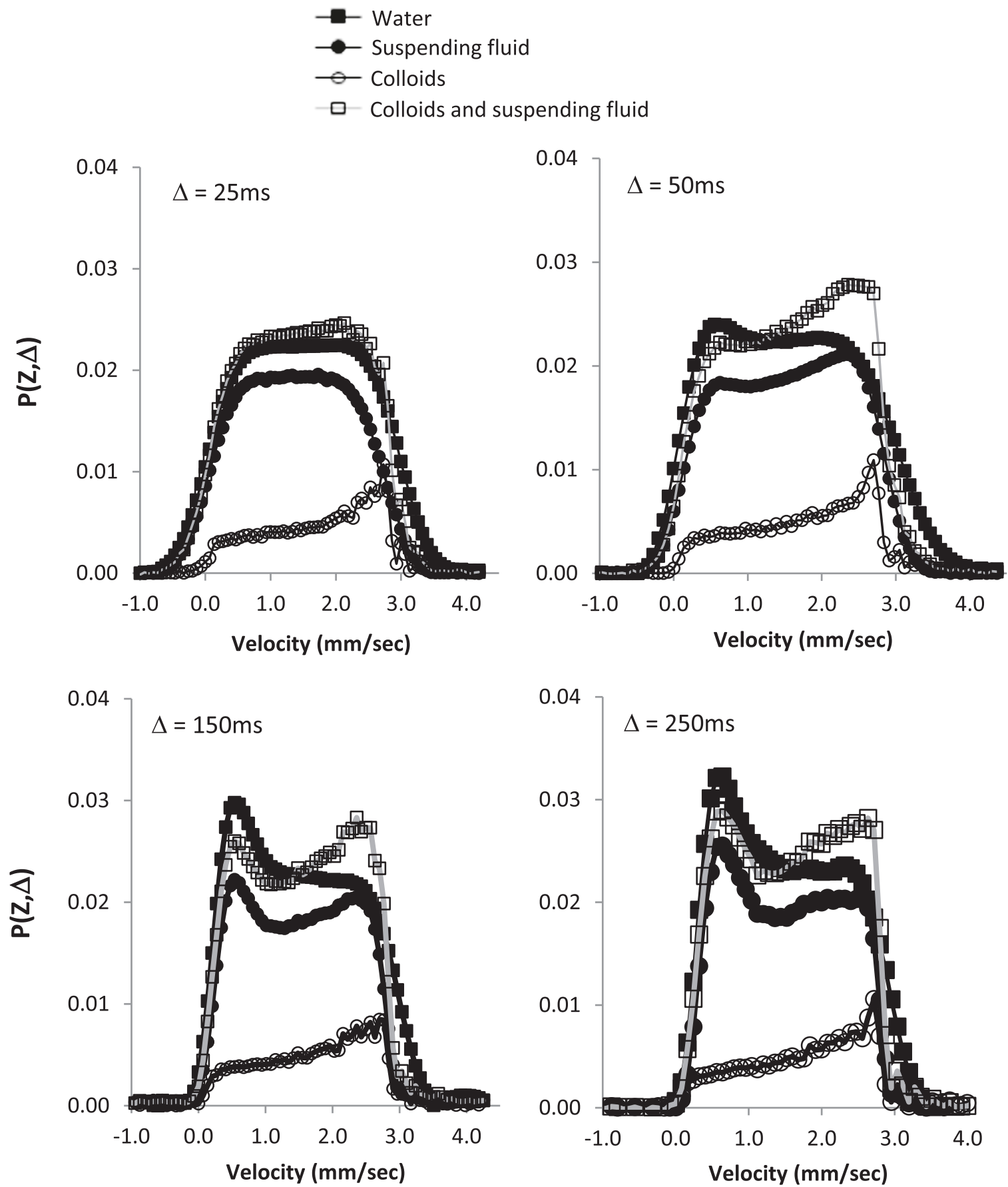

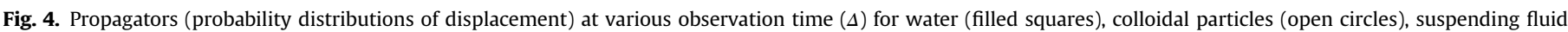

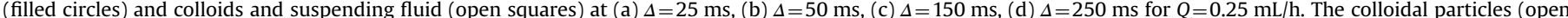

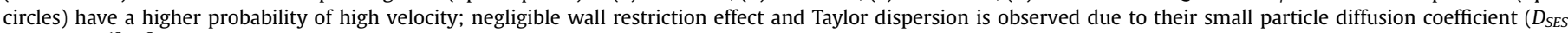

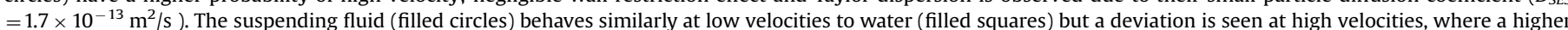

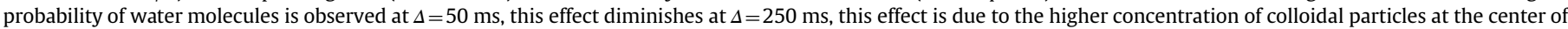
the capillary. The propagators are normalized such that $\int P_{\text {water }} d R=\int P_{c S} d R=1$, where $P_{c S}$ is defined by Eq. $(9), \int P_{S} d R=1-\phi, \int P_{c} d R=\phi$.

The net phase shift imposed on the spins by the pair of gradient pulses is

$\phi(\mathbf{r})=\gamma \delta \mathbf{g} \cdot\left(\mathbf{r}^{\prime}-\mathbf{r}\right)=\gamma \delta \mathbf{g}\langle\mathbf{v}\rangle \Delta$

and is directly proportional to the molecular displacement or mean velocity $\langle\mathbf{v}\rangle=\frac{\left(\mathbf{r}^{\prime}-\mathbf{r}\right)}{\Delta}$. For the ensemble of molecules within the $\mu$-capillary the total NMR signal acquired has the form,

$E(\mathbf{g}, \delta, \Delta)=\frac{S(\mathbf{g}, \delta, \Delta)}{S(0, \delta, \Delta)}=\int \rho(\mathbf{r}) \int P_{i}\left(\mathbf{r} \mid \mathbf{r}^{\prime}, \Delta\right) \exp \left[i \gamma \delta g \cdot\left(\mathbf{r}^{\prime}-\mathbf{r}\right)\right] \mathbf{d r} \mathbf{r}^{\prime} \mathbf{d r}$ where $\rho(\mathbf{r})$ is the spin density proportional to the molecular density of the sample. As negligible motion occurs during gradient phasing during $\delta$ the so-called narrow pulse approximation $(\delta<<\Delta)$ is normally applicable. The total signal is a superposition of transverse magnetization, in which each phase term $\exp \left[i \gamma \delta g \cdot\left(\mathbf{r}^{\prime}-\mathbf{r}\right)\right]$ is weighted by the probability of a molecule, originally located at $\mathbf{r}$, moving to $\mathbf{r}^{\prime}$ in the time $\Delta$, i.e. $\rho(\mathbf{r}) P_{i}\left(\mathbf{r} \mid \mathbf{r}^{\prime}, \Delta\right)$. Introducing a displacement $\mathbf{R}=\mathbf{r}^{\prime}-\mathbf{r}$ and taking the ensemble average over all spins, $\int \rho(\boldsymbol{r}) P_{i}(\boldsymbol{r} \mid \boldsymbol{r}+\boldsymbol{R}, \Delta) d \boldsymbol{r}$ yields the average 


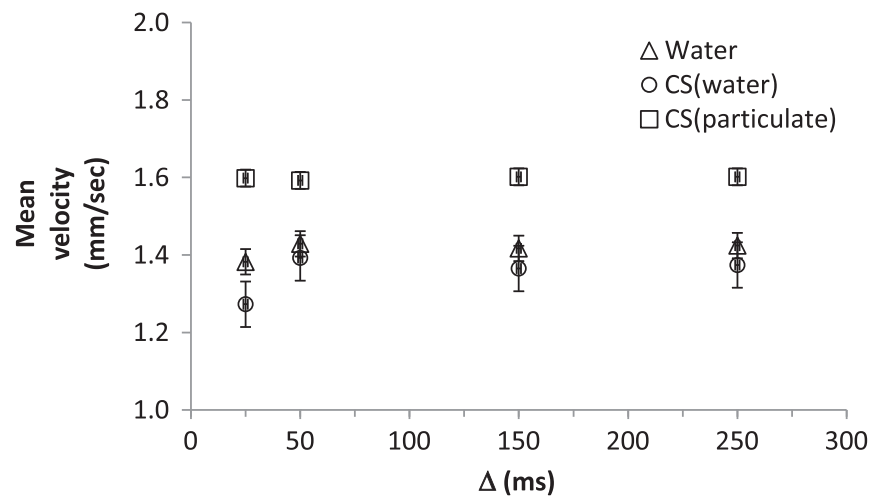

Fig. 5. The mean velocities for $Q=0.25 \mathrm{~mL} / \mathrm{h}$ as function of observation time $(\Delta)$ for the suspending fluid and particulates for the colloidal suspension (CS). For comparison are the mean velocities of water (open triangle) flowing in the $\mu$-capillary. The mean velocity for the water averaged over $\Delta$ is $1.41 \pm 0.02 \mathrm{~mm} / \mathrm{s}$, while for the combined CS solution it is $1.40 \pm 0.05 \mathrm{~mm} / \mathrm{s}$. These results demonstrate the effect of higher concentration of particulates in the center of the capillary versus closer to the capillary wall.

propagator, $P(\mathbf{R}, \Delta)$. By introducing a $\mathbf{q}$-space vector, $\mathbf{q}=\frac{\gamma \delta \mathbf{g}}{2 \pi}$ which is the Fourier reciprocal wavelength of displacement, the dynamic analog of the static reciprocal space i.e. $\mathbf{k}$-space vector (Blumich 2000, Callaghan 1991), Eq. (7) can be re-written as,

$E(\mathbf{q}, \Delta)=\int P_{i}(\mathbf{R}, \Delta) \exp [i 2 \pi \mathbf{q} \cdot \mathbf{R}] \mathbf{d} \mathbf{R}$

This shows that $P(\mathbf{R}, \Delta)$ is obtained from a Fourier transform of $E(\mathbf{q}, \Delta)$ with respect to $\mathbf{q}$. The $\mathbf{q}$-space pulse sequences discussed above to measure displacement can be combined with NMR imaging methods to obtain an image where each pixel has q-space information, allowing velocity maps to be obtained. The basics of NMR imaging (MRI) can be sourced from the vast MRI literature (Blumich, 2000; Callaghan, 1991; Liang and Lauterbur, 2000) and a standard technique of spin warp imaging is used in this work as the basis for sampling the spatial domain k-space. The combined $\mathbf{k}$-space and $\mathbf{q}$-space pulse sequence to obtain velocity maps is shown in Fig. 1b - NMR parameters are shown in caption.

An important step before dynamic experiments on the colloidal dispersions are conducted is the use of NMR imaging experiments to verify the alignment with the applied magnetic field $B_{0}(z$-direction) of the capillary inside the rf coil. Experiments on water flow through the capillary using the same NMR pulse sequences were conducted for a baseline comparison to the suspension flows.

The colloidal suspensions were prepared using an evaporative emulsification procedure detailed by Loxley and Vincent (1998). The resulting volume fraction and particle size were $\phi=0.22$ and $a=1.25 \mu \mathrm{m}$. The experimental setup (Fig. 2a) consists of a syringe pump (22 Harvard Science ${ }^{\circledR}$ ) with a $5 \mathrm{~mL}$ glass syringe (BD Medical ${ }^{\circledR}$ ) pumping the fluid through $2 \mathrm{~m}$ of $1 \mathrm{~mm}$ internal diameter (ID) polytetrafluoroethylene (PTFE) tubing (GE Healthcare ${ }^{\circledR}$ ) which is mated to a $50 \mathrm{~cm}$ long $252 \mu \mathrm{m}$ ID capillary (Polymicro ${ }^{\circledR}$ ) by epoxy. The microcapillary opens into another PTFE tubing ( $2.5 \mathrm{~m}$ of $1 \mathrm{~mm}$ ID), which is also mated to it by epoxy. To stabilize the $\mu$-capillary inside the rf coil it is threaded through a $1 \mathrm{~mm}$ i.d. glass capillary and a $5 \mathrm{~mm}$ i.d. NMR tube sealed at each end by flexible silicone tubing forming a rigid structure. At the outlet of this flow setup is a graduated cylinder to collect the effluent. The concentration of the effluent is tested using a micro-centrifuge to determine when steady state in effluent particle concentration is reached. Fig. 2b shows an example of a NMR frequency spectrum showing distinct frequencies for the water suspending fluid phase and oil particle phase components of the colloidal suspension. These spectral components can be separately probed using the PGSE NMR method discussed above to determine the transport dynamics of each phase in the colloidal suspensions simultaneously within the same sample. The particles were characterized using field emission microscopy (FEM) and light microscopy (Fig. 2c) techniques to determine particle shape, smoothness and size distribution. For the colloidal suspensions used in this work $D_{S E S}=1.7 \times 10^{-13} \mathrm{~m}^{2} / \mathrm{s}$.

\section{Results and discussion}

Fig. 3 shows a series of propagators obtained for water flowing at $Q=0.25 \mathrm{~mL} / \mathrm{h}$ for $\Delta=25,50,150,250$ and $500 \mathrm{~ms}$ with displacement measured in the axial-flow $(z)$ direction. These show a narrowing in the probability distribution of displacement with increasing observation time $\Delta$. The initial propagator $(\Delta=25 \mathrm{~ms})$ has the shape of a Heaviside-hat function convoluted with a Gaussian function, corresponding to Poiseuille flow combined with diffusion (Codd et al. 1999). As displacement time increases $(\Delta \rightarrow 500 \mathrm{~ms})$ a higher probability hump appears for water molecules experiencing small displacements i.e. close to the wall due to the wall reflections. At the largest observation time $(\Delta=500 \mathrm{~ms})$ the propagator is still evolving towards the expected Gaussianshaped probability distribution due to Taylor dispersion (Codd et al. 1999). The systems studied in this work are in the preasymptotic hydrodynamic dispersion regime (i.e. $\Delta<<R^{2} / D_{0}$, for water: $R^{2} / D_{0}=7.2 \mathrm{~s}$ with $D_{0}=2.2 \times 10^{-9} \mathrm{~m}^{2} / \mathrm{s}$, for colloidal particles: $R^{2} / D_{S E S}=23.1 \mathrm{~h}$ with $D_{S E S}=1.7 \times 10^{-13} \mathrm{~m}^{2} / \mathrm{s}$ (Fridjonsson et al. 2014b)).

Fig. 4 shows a series of spectrally resolved propagators at $Q=0.25 \mathrm{~mL} / \mathrm{h}$ for $\Delta=25,50,150$ and $250 \mathrm{~ms}$ for a water propagator, the suspension colloidal particles propagator, the suspending fluid propagator and combined propagator $\left(P_{c s}\right)$ which is defined as

$P_{C S}(r)=(1-\phi) P_{S}(r)+\phi P_{C}(r)$

Where $\phi$ is the particle volume fraction $(\phi=0.22), P_{s}(r)$ is the propagator of the suspending fluid and $P_{c}(r)$ is the propagator of the colloidal particles. The particle propagator clearly shows that the colloids have a higher probability of occupying regions of high displacement, i.e. the center of the capillary, than regions of low displacement (i.e. close to wall). The colloids are not affected by Taylor dispersion on the time scales being investigated, $\Delta=25 \mathrm{~ms}$ to $500 \mathrm{~ms}$, which causes their probability distribution to remain approximately constant with time, while the suspending fluid which is affected by Taylor dispersion experiences a narrowing of its distribution with time, akin to what is seen for water (Fig. 3). Due to wall reflections suspending fluid molecules have an increased probability of experiencing small displacement, while at higher displacements a region of high local probability is seen for $\Delta=50 \mathrm{~ms}$ (Fig. 4), this appears to progress to a similarly shaped distribution as seen for water by $\Delta=250 \mathrm{~ms}$ (Fig. 4). This dynamic behavior in the suspending fluid is due to restricted diffusion caused by a higher concentration of colloidal particles occupying the central region of the microcapillary. For the colloidal particle propagators Gibbs ringing (Amartur and Haacke 1991), a Fourier transformation truncation artifact, can be observed which occurs due to an abrupt change in particle concentration at the highest velocities, although interesting due to its relationship to the blunting of the velocity profile, this does not significantly affect the analysis conducted in this work as it can be corrected for.

By measuring the NMR signal from the particulate and suspending fluid phases unambiguous results can be obtained about the relative velocities of each phase, and these can be compared using Eq. (9). The results for the mean velocities of each phase at different $\Delta$ are shown in Fig. 5 . The particulate phase has a mean 
(A)
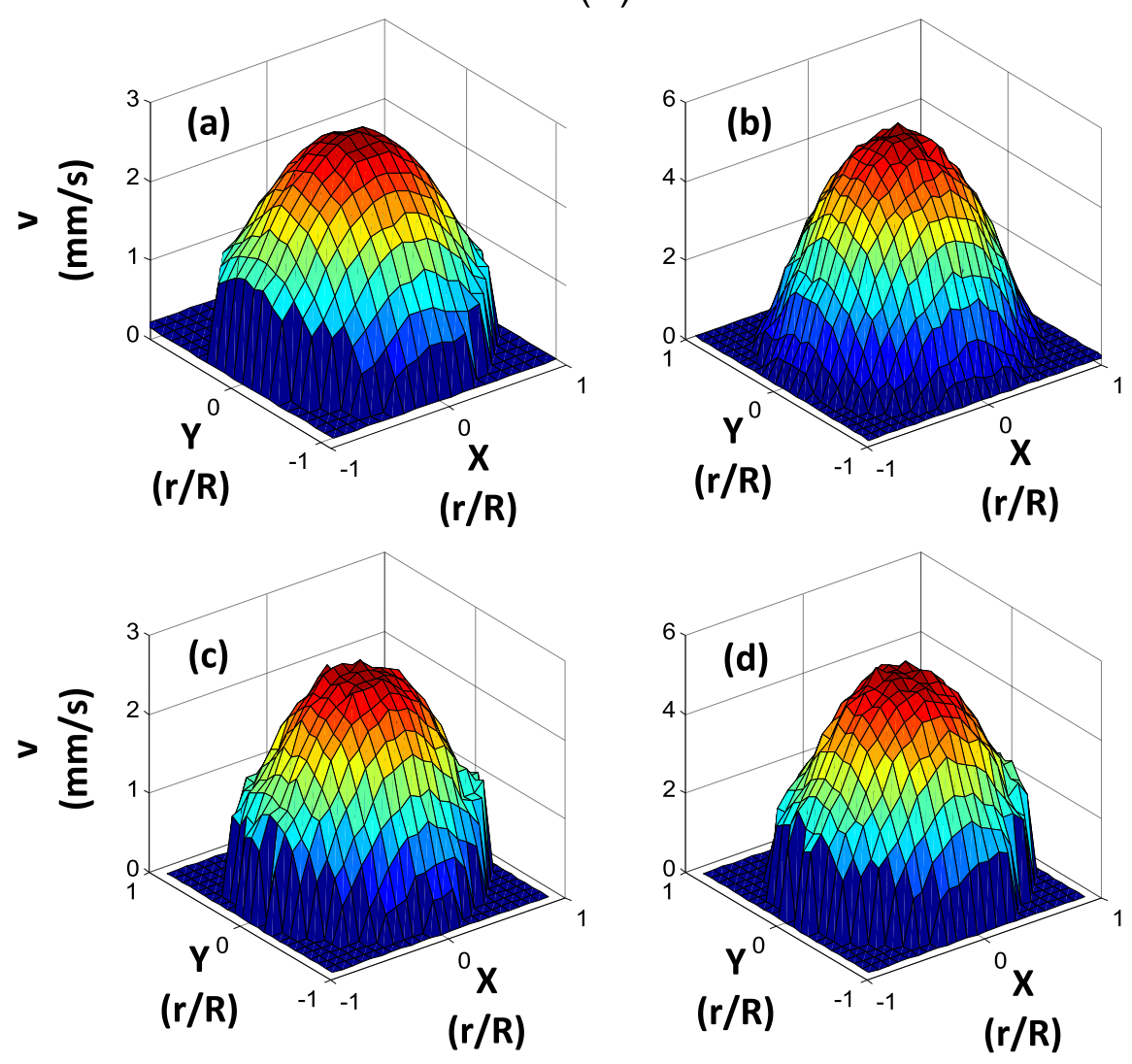

(B)
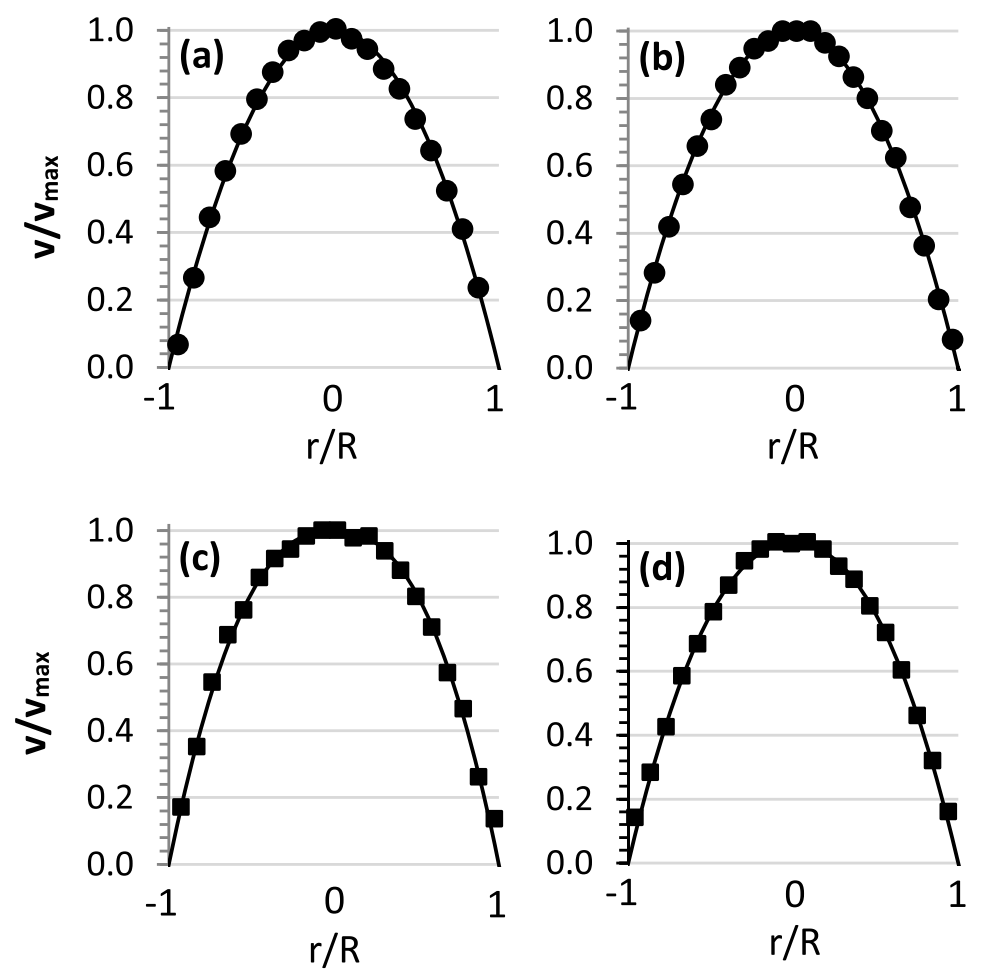

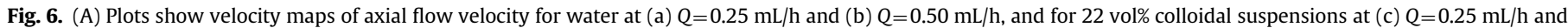

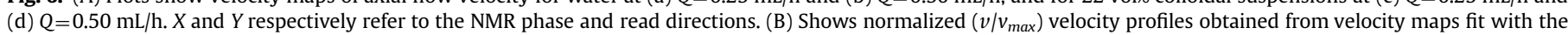

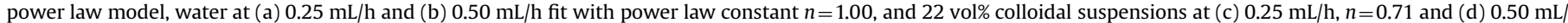
h, $n=0.85$. 

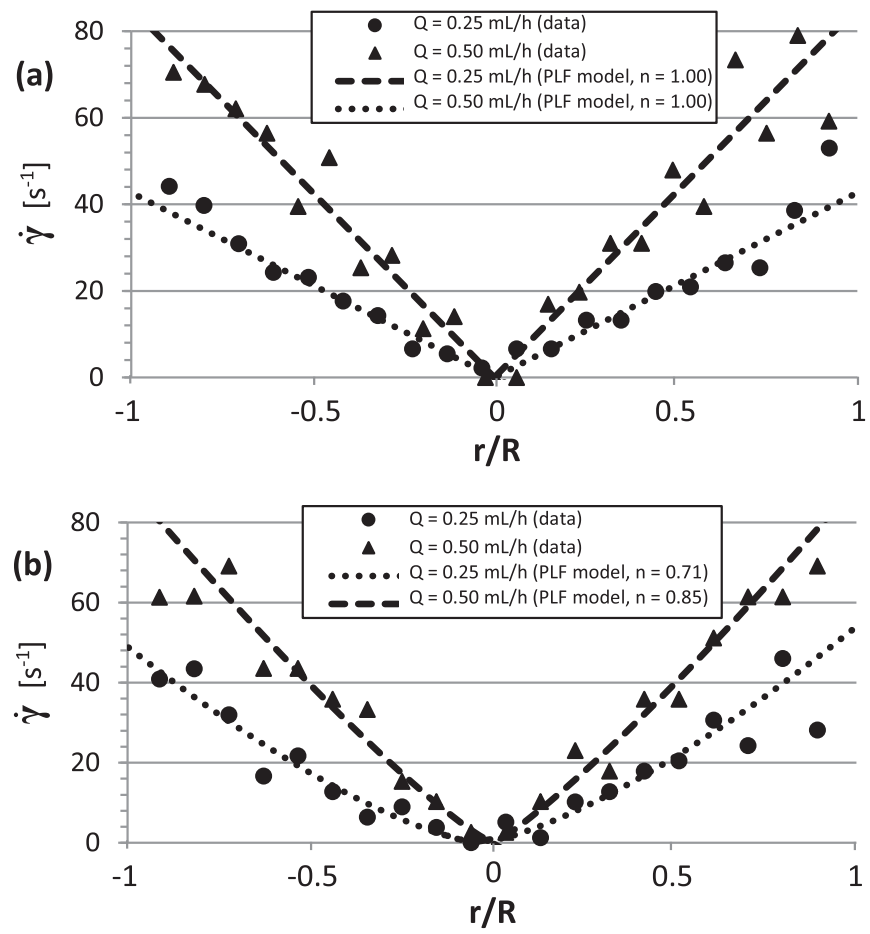

Fig. 7. Measured shear rates $(\dot{\gamma})$ derived from the velocity profiles obtained from velocity maps for $Q=0.25 \mathrm{~mL} / \mathrm{h}$ and $0.50 \mathrm{~mL} / \mathrm{h}$, fit with power law model (a) shows results for water and (b) shows results for $22 \mathrm{vol} \%$ colloidal suspensions. A power law constant of $n=1$ fits the water data well, while a power law constant of $n<1$ (or shear thinning) fits the $22 \mathrm{vol} \%$ colloidal suspension data well.

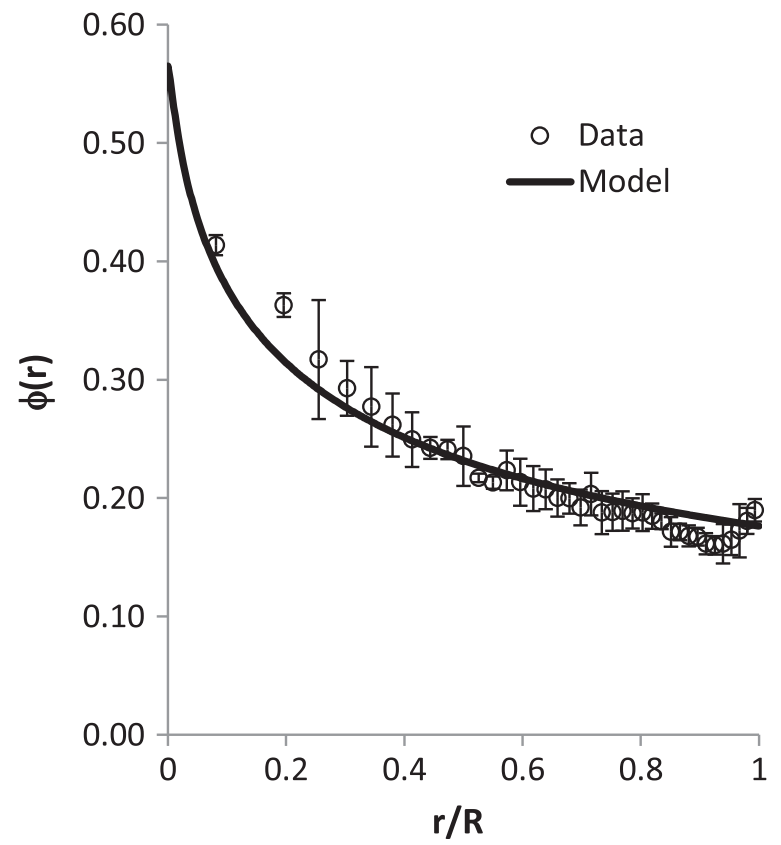

Fig. 8. The colloid volume fraction $\phi$ as a function of radial position $(r / R)$ obtained from propagators and velocity maps at $Q=0.25 \mathrm{~mL} / \mathrm{h}$, the solid lines are for model of shear induced migration for $P e_{p}=270$ (Frank et al., 2003). When data is compared with the model a deviation at the center of the capillary is evident due to the un-physical cusping of the model, while at the capillary wall a slightly higher particle concentration is evident.

velocity of $\langle v\rangle_{c}=1.60 \pm 0.01 \mathrm{~mm} / \mathrm{s}$ while the suspending fluid phase has a mean velocity of $\langle v\rangle_{s}=1.34 \pm 0.05 \mathrm{~mm} / \mathrm{s}$. These can be used in conjunction with Eq. (9) to calculate the colloidal suspension velocity $\langle v\rangle_{c s}=1.40 \pm 0.06 \mathrm{~mm} / \mathrm{s}$ which compares favorably with the measured velocity for water $\langle v\rangle_{w}=1.41 \pm 0.02 \mathrm{~mm} / \mathrm{s}$.
These results show that the mean velocity of the colloidal particles is greater than the suspending fluid causing important radially dependent deviations in the local $\mu$-rheology within the capillary. These results emphasize the importance of determining the effect of shear induced particle migration in a microcapillary, as this is critical to the correct functionality of microfluidic devices.

To further investigate these phenomena velocity maps were obtained for water and $22 \mathrm{vol} \%$ colloidal suspension flowing at $Q=0.25$ and $0.50 \mathrm{~mL} / \mathrm{h}$, see Fig. $6 \mathrm{~A}$. These velocity maps have a $10 \mathrm{~mm}$ slice thickness with $11.7 \times 11.7 \mu \mathrm{m}^{2}$ in-plane spatial resolution. To characterize the rheology, velocity profiles were extracted from the velocity maps (see Fig. 6A) and fit using a power law fluid model (Bird et al. 1987),

$v_{z}(r)=v_{z, \max }\left[1-\left(\frac{r}{R}\right)^{\frac{1}{n}+1}\right]$

where $v_{z, \max }$ is the maximum velocity in the axial flow $(z)$ direction, $r$ is the radial position within the capillary and $n$ is the power law fluid exponent. For a shear thinning fluid $n<1$, for a Newtonian fluid $n=1$ and a shear thickening fluid $n>1$. The data is fit using the method of least squares. The velocity profile for water is best fit with $n=1$, validating the procedure for the known Newtonian rheology of water flowing in the microcapillary. The $22 \mathrm{vol} \%$ colloidal suspension at the two flow rates shown have best fits $n=0.71$ and $n=0.85$, indicating a non-Newtonian shear thinning rheology with shear rate dependent power law index. The maximum velocities $\left(v_{z, \max }\right)$ for the colloidal suspensions when normalized with those obtained for water are respectively $0.87 v_{z, \text { max,water }}$ and $0.96 v_{z, \text { max,water }}$ for $Q=0.25$ and $0.50 \mathrm{~mL} / \mathrm{h}$. The variation in the power law fluid exponent with flow is due to shear dependent microstructural regime differences and demonstrates the limitations of using a simple power law model.

To further characterise the macroscopic rheology, shear rates were extracted from the velocity profiles using $\dot{\gamma}=\frac{\Delta v_{z}(r)}{\Delta r}=\frac{v_{z, i}-v_{z, i-1}}{r_{i}-r_{i-1}}$ (Fig. 7). The results explicitly demonstrate the potential of NMR as a measurement tool for determining the spatial distribution of shear rates inside a microcapillary. A main source of deviation from Newtonian behavior in the microcapillary is due to shear induced migration (Brown et al., 2009, Semwogerere et al., 2007, Yurkovetsky and Morris, 2008).

The particle concentration profiles as a function of radial position (Fig. 8) are extracted from the propagators and the velocity maps, i.e. the propagators give the concentration at a certain displacement (colloid NMR signal versus the suspending fluid NMR signal) while the velocity map allows for these displacements to be assigned to a radial position. The detailed procedure to obtain the concentration profile is outlined in Brown et al. (2009). In Brown et al. (2009) it was also shown that the combined signal of oil and water phases matched the expected velocity profile in the capillary, demonstrating no velocity slip within MRI voxels. A normal stress difference for the steady state migration model based on the work of Morris et al. (Frank et al., 2003, Morris and Boulay, 1999, Semwogerere et al., 2007) is fit to the data for $P e_{p}=270$. While there is an ongoing debate on the theoretical limits of the suspension balance model (Nott et al., 2011), it is in good agreement with the acquired data. The model generally captures the radial particle concentration profile observed in the data, however it can be seen that this model deviates from the data both close to the wall $(r / R>0.75)$ and in the center of the microcapillary $(r / R<0.25)$. At the center of the microcapillary the model allows for non-physical cusping (Frank et al., 2003), in the microcapillary as $\phi$ increases there is also an increase in particleparticle interactions which generates a barrier to high $\phi$ values. The effect of particle-particle interactions is to decrease the 
effective diffusion or particle mobility as the particles get closer to the center, which should counteract the particle-migration driving force. This effect explains the distribution in the concentration profile close to the center. Interestingly, closest to the wall, corresponding to $r / R=0.96-1.00$ or $\sim 5 \mu \mathrm{m}, \phi$ increases with increasing $r / R$. Recent work on the dynamics of concentrated hardsphere colloids near a wall by Michailidou et al. (2009) showed that the hydrodynamic drag that slows a particle in the vicinity of the wall is progressively weakened at high $\phi$ due to a counterbalance by hydrodynamic interactions. This work conducted at $P e_{p}=0$ indicates that for approximately $\phi \geq 0.1$ this shielding of the wall effect should become significant, while once above $\phi \sim 0.35$ diffusion in bulk or close to the wall are indistinguishable. The results shown in Fig. 8 and discussed above show the strong dependence of $\phi$ with radial position. Brady and Vicic (1995) have shown by simulation that the onset of non-Newtonian behavior in a hard sphere colloidal suspension is manifest in normal stress differences. These stress differences have been used (Brown et al., 2009, Frank et al., 2003, Semwogerere et al., 2007) to explain the observed migration of colloidal particles leading to more concentrated regions where lower shear rates exists. The current work demonstrates the importance of these effects in a microcapillary. Furthermore, verification of recent theoretical developments regarding Taylor dispersion of particles in suspensions flows in capillaries by Griffiths and Stone (2012) and Ramachandran (2013) provides impetus for future use of the techniques presented in this work.

\section{Conclusions}

This work has demonstrated a useful experimental framework for the simultaneous interrogation of shear rheology of the suspending fluid and particle phases of colloidal suspensions inside a microcapillary using dynamic NMR techniques. The dynamic behavior of the suspending fluid has been shown to carry within it information about the structure of the colloidal particle ensembles on the time scales investigated $(\Delta=25 \mathrm{~ms} \rightarrow 250 \mathrm{~ms})$ providing detailed experimental data for further investigation and model verification. The importance of determining the particle concentration profile within microcapillaries is explicitly demonstrated as shear induced migration causes significant concentration gradients to occur at strong flow conditions (i.e. $P e_{p}=270$ ) and impacts effective rheology.

\section{Acknowledgments}

JDS acknowledges funding from NSF CAREER Award CTS0348076. The M.J. Murdock. Charitable Trust and NSF MRI program provided equipment funding. EOF acknowledges support from University of Western Australia (UWA) and Research Collaboration Award (RCA).

\section{References}

Amartur, S., Haacke, E.M., 1991. Modified iterative model based on data extrapolation method to reduce Gibbs ringing. J. Magn. Reson. Imaging 1 (3), 307-317.

Antl, L., Goodwin, J.W., Hill, R.D., Ottewill, R.H., Owens, S.M., Papworth, S., Waters, J. A., 1986. The preparation of poly(methyl methacrylate) lattices in nonaqueous media. Colloids Surf. 17 (1), 67-78.

Beatus, T., Bar-Ziv, R., Tlusty, T., 2007. Anomalous microfluidic phonons induced by the interplay of hydrodynamic screening and incompressibility. Phys. Rev. Lett. 99 (12), 124502

Bergenholtz, J., Brady, J.F., Vicic, M., 2002. The non-Newtonian rheology of dilute colloidal suspensions. J. Fluid Mech. 456, 239-275.
Bird, R.B., Armstrong, R.C., Hassager, O., 1987. Dynamics of Polymeric Liquids Fluid Dynamics. 1. Wiley Interscience, New York.

Blumich, B., 2000. NMR Imaging of Materials. Oxford university press, Oxford.

Brady, J.F., 1993. The rheological behavior of concentrated colloidal dispersions. J. Chem. Phys. 99 (1), 567-581.

Brady, J.F., 1996. Model hard-sphere dispersions: Statistical mechanical theory, simulations, and experiments. Curr. Opin. Colloid Interface Sci. 1 (4), 472-480.

Brady, J.F., Vicic, M., 1995. Normal stresses in colloidal dispersions. J. Rheol. 39 (3) $545-566$.

Brosten, T.R., Fridjonsson, E.O., Codd, S.L., Seymour, J.D., 2010. NMR measurement of the transport dynamics of colloidal particles in an open cell polymer foam porous media. J. Colloid Interface Sci. 349 (1), 384-391.

Brown, J.R., Fridjonsson, E.O., Seymour, J.D., Codd, S.L., 2009. Nuclear magnetic resonance measurement of shear-induced particle migration in Brownian suspensions. Phys. Fluids 21, 093301.

Brown, J.R., Seymour, J.D., Codd, S.L, Fridjonsson, E.O., Cokelet, G.R., Nydén, M. 2007. Dynamics of the solid and liquid phases in dilute sheared Brownian suspensions: irreversibility and particle migration. Phys. Rev. Lett. 99 (24) 240602.

Brown, R., 1828. A brief account of microscopical observations made in the months of June, July and August, 1827, on the particles contained in the pollen of plants and on the general existence of active molecules in organic and inorganic bodies. Philos. Mag. 4, 161-173.

Callaghan, P.T., 2011. Translational Dynamics and Magnetic Resonance: Principles of Pulsed Gradient Spin Echo NMR. Oxford University Press, Oxford.

Callaghan, P.T., 1991. Principles of Nuclear Magnetic Resonance Microscopy. Oxford University Press, Oxford.

Chelakkot, R., Winkler, R.G., Gompper, G., 2012. Flow-Induced Helical Coiling of Semiflexible Polymers in Structured Microchannels. Phys. Rev. Lett. 109 (17) 178101.

Christopher, G.F., Anna, S.L., 2007. Microfluidic methods for generating continuous droplet streams. J. Phys. D: Appl. Phys. 40 (19), R319.

Codd, S.L., Manz, B., Seymour, J.D., Callaghan, P.T., 1999. Taylor dispersion and molecular displacements in Poiseuille flow. Phys. Rev. E 60 (4), R3491-R3494.

Di Carlo, D., Edd, J.F., Humphry, K.J., Stone, H.A., Toner, M., 2009. Particle segregation and dynamics in confined flows. Phys. Rev. Lett. 102 (9), 094503.

Einstein, A., 1906. The theory of the Brownian motion. Ann. Phys. 19 (2), 371-381.

Evertz, L.Q., Rassi, E.M., Kennedy, J.R., Codd, S.L., Seymour, J.D., 2012. Oscillatory flow phenomena in simple and complex fluids. Appl. Magn. Reson. 42 (2) 211-225.

Foss, D.R., Brady, J.F., 2000. Structure, diffusion and rheology of Brownian suspensions by Stokesian dynamics simulation. J. Fluid Mech. 407, 167-200.

Frank, M., Anderson, D., Weeks, E.R., Morris, J.F., 2003. Particle migration in pressure-driven flow of a Brownian suspension. J. Fluid Mech. 493, 363-378.

Fridjonsson, E.O., Codd, S.L., Seymour, J.D., 2014a. Application of PFG-NMR to study the impact of colloidal deposition on hydrodynamic dispersion in a porous medium. Transp. Porous Media, 1-14.

Fridjonsson, E.O., Seymour, J.D., Codd, S.L., 2014b. Anomalous preasymptotic colloid transport by hydrodynamic dispersion in microfluidic capillary flow. Phys. Rev. E 90 (1), 010301.

Fridjonsson, E.O., Seymour, J.D., Cokelet, G.R., Codd, S.L., 2011. Dynamic NMR microscopy measurement of the dynamics and flow partitioning of colloidal particles in a bifurcation. Exp. Fluids 50 (5), 1335-1347.

Griffiths, I.M., Stone, H.A., 2012. Axial dispersion via shear-enhanced diffusion in colloidal suspensions. EPL 97 (5), 58005.

Harasim, M., Wunderlich, B., Peleg, O., Kröger, M., Bausch, A.R., 2013. Direct observation of the dynamics of semiflexible polymers in shear flow. Phys. Rev. Lett. 110 (10), 108302.

Janssen, P.J.A., Baron, M.D., Anderson, P.D., Blawzdziewicz, J., Loewenberg, M., Wajnryb, E., 2012. Collective dynamics of confined rigid spheres and deformable drops. Soft Matter 8 (28), 7495-7506.

Leal, L.G., 1980. Particle motions in a viscous fluid. Annu. Rev. Fluid Mech. 12 (1), 435-476.

Liang, Z.-P., Lauterbur, P.C., 2000. Principles of Magnetic Resonance Imaging. IEEE Press, New York.

Loxley, A., Vincent, B., 1998. Preparation of poly (methylmethacrylate) microcapsules with liquid cores. J. Colloid Interface Sci. 208 (1), 49-62.

Michailidou, V.N., Petekidis, G., Swan, J.W., Brady, J.F., 2009. Dynamics of concentrated hard-sphere colloids near a wall. Phys. Rev. Lett. 102 (6), 4.

Morris, J.F., Boulay, F., 1999. Curvilinear flows of noncolloidal suspensions: the role of normal stresses. J. Rheol. 43 (5), 1213-1237.

Nott, P.R., Guazzelli, E., Pouliquen, O., 2011. The suspension balance model revisited. Phys. Fluids 23 (4), 043304

Pine, D.J., Gollub, J.P., Brady, J.F., Leshansky, A.M., 2005. Chaos and threshold for irreversibility in sheared suspensions. Nature 438 (7070), 997-1000.

Price, W.S., 2009. NMR Studies of Translational Motion: Principles and Applications Cambridge University Press, Cambridge.

Ramachandran, A., 2013. A macrotransport equation for the particle distribution in the flow of a concentrated, non-colloidal suspension through a circular tube. J. Fluid Mech. 734, 219-252.

Reddig, S., Stark, H., 2013. Nonlinear dynamics of spherical particles in Poiseuille flow under creeping-flow condition. J. Chem. Phys. 138 (23), 234902.

Russel, W.B., 1981. Brownian motion of small particles suspended in liquids. Annu. Rev. Fluid Mech. 13, 425-455.

Russel, W.B., Saville, D.A., Schowalter, W.R., 1989. Colloidal Dispersions. Cambridge University Press, Cambridge, UK. 
Segre, G., Silberberg, A., 1962. Behaviour of macroscopic rigid spheres in Poiseuille flow Part 2. Experimental results and interpretation. J. Fluid Mech. 14 (1), 136-157.

Semwogerere, D., Morris, J.F., Weeks, E.R., 2007. Development of particle migration in pressure-driven flow of a Brownian suspension. J. Fluid Mech. 581, 437-451.

Smoluchowski, M., 1906. Zur kinetischen Theorie der Brownschen Molekularbewegung und der suspensionen. Ann. Phys. 21, 765-780.

Snijkers, F., Pasquino, R., Vermant, J., 2013. Hydrodynamic interactions between two equally sized spheres in viscoelastic fluids in shear flow. Langmuir 29 (19), 5701-5713.

Sochol, R.D., Casavant, B.P., Dueck, M.E., Lee, L.P., Lin, L., 2011. A dynamic bead-based microarray for parallel DNA detection. J. Micromech. Microeng. 21 (5), 054019.

Stickel, J.J., Powell, R.L., 2005. Fluid mechanics and rheology of dense suspensions. Annu. Rev. Fluid Mech. 37, 129-149.

Stone, H.A., Stroock, A.D., Ajdari, A., 2004. Engineering flows in small devices: microfluidics toward a lab-on-a-chip. Annu. Rev, Fluid Mech. 36, 381-411.

Subramanian, G., Koch, D.L., 2006. Inertial effects on the transfer of heat or mass from neutrally buoyant spheres in a steady linear velocity field. Phys. Fluids 18 (7), 073302.

Vermant, J., Solomon, M.J., 2005. Flow-induced structure in colloidal suspensions. J. Phys.- Condens. Matter 17 (4), R187-R216.

Wassenius, H., Callaghan, P.T., 2004. Nanoscale NMR velocimetry by means of slowly diffusing tracer particles. J. Magn. Reson. 169 (2), 250-256.

Wassenius, H., Callaghan, P.T., 2005. NMR velocimetry studies of the steady-shear rheology of a concentrated hard-sphere colloidal system. Eur. Phys. J. E 18 (1), 69-84.

Wassenius, H., Nyden, M., Vincent, B., 2003. NMR diffusion studies of translational properties of oil inside core-shell latex particles. J. Colloid Interface Sci. 264 (2), 538-547.

Yun, H., Kim, K., Lee, W.G., 2013. Cell manipulation in microfluidics. Biofabrication 5 (2), 022001.

Yurkovetsky, Y., Morris, J.F., 2008. Particle pressure in sheared Brownian suspensions. J. Rheol. 52 (1), 141-164. 